\title{
On the 9th International Conference "Numerical Geometry, Construction of Numerical Grids and High-Performance Computations (NUMGRID 2018/Voronoi 150)"
}

\author{
V. Garanzha, L. Kamenski, and Hang Si \\ Received February 18, 2019; revised February 18, 2019; accepted August 5, 2019
}

DOI: $10.1134 / \mathrm{S} 0965542519120170$

This issue presents papers based on talks given at the 9th International Conference "Numerical Geometry, Construction of Numerical Grids, and High-Performance Computations (NUMGRID 2018/Voronoi 150)," which was dedicated to Georgy Voronoi on the occasion of his 150th birthday. The proceedings of the NUMGRID2018/Voronoi 150 Conference were published by Springer in Lecture Notes in Computational Science and Engineering series in 2019. In its foreword, Stefano Paoletti (Siemens SISW, Rome, Italy), a world leading expert in the field of polyhedral mesh generation noted that "after century and a half, Voronoi's work is still the base of many researchers and has received a renovated attention, in particular, in the field of mesh generation. Voronoi graph and its dual, the Delaunay tessellation, are the base for the most popular and effective tetrahedral and polyhedral mesh generators.

In addition, recently a number of researchers have also attempted a direct usage of the Voronoi graph ideas to construct a polyhedral mesh, avoiding the usage of the Delaunay tessellation. Mesh optimization is also greatly affected by concepts that have their foundation in Voronoi's work.

Most academic and industrial simulation packages offer, one way or another, a mesh generator that exploits the power of Voronoi graph. This should make us consider how the advances in mathematics, achieved in "era" when computers were not yet available, continue to affect our life with unexpected fruits.

Computational Fluid Dynamics and Stress Analysis, the two main areas where simulation and therefore mesh generation play a strong role, are responsible for reduced cost and time-to-market of many objects that we commonly use in our day-to-day life. Cars, airplanes, engines, turbomachines, ships, roads, bridges, buildings, etc. can now be designed and tested at a pace that was unreachable only a few decades ago.

It is worth noting that Voronoi graph has been used in other research and application areas such as medicine, chemistry, biology, logistics, and operations research as a demonstration that a seminal idea can in time generate very fruitful consequences..."

The conference was held at the Dorodnitsyn Computing Center of the Russian Academy of Sciences, Moscow, from December 3 to 5, 2018. Recall that the NUMGRID conference is held every 2 years, starting in 2002, when it was organized for the first time by the well-known Soviet and Russian expert in mesh generation Sergei Aleksandrovich Ivanenko, who tragically died in 2003, and by Vladimir Anatol'evich Garanzha. At present, this conference has become widely known and has earned international recognition. The main issue addressed by the conference is associated with mesh generation methods, which are of crucial importance for numerical simulation in all subject areas. Mesh generation is a multidisciplinary field intersecting mathematics, computer science, and engineering. The goal of the conference and of this issue was to provide a good balance between engineering algorithms and their mathematical justification. The issue contains papers covering the state of the art in numerical geometry, mesh generation and adaptation, development of algorithms and applied software, and various applications. The material is focused on the theory and algorithms for analysis and construction of Delaunay-Voronoi partitions, as well as on mesh optimization and deformation, the uniform distribution principle, error analysis, discrete differential geometry, duality in mathematical programming and numerical geometry, grid methods for optimization and optimal control, iterative solvers for variational problems, and the design of algorithms and application software. Applications of the described methods include mathematics, physics of the solid state, biology, medicine, and engineering. 